\title{
Phase Estimation for Coherent Optical OFDM
}

\author{
Xingwen Yi, William Shieh, and Yan Tang
}

\begin{abstract}
Phase estimation is one of the enabling functionalities in coherent optical orthogonal frequency-division-multiplexing (CO-OFDM) receivers. In this letter, we compare pilot-aided and data-aided phase estimation methods for a CO-OFDM transmission experiment at $8 \mathrm{~Gb} / \mathrm{s}$ over $1000-\mathrm{km}$ standard single-mode fiber without optical dispersion compensation. We also show that as few as five subcarriers are sufficient for pilot-aided phase estimation.
\end{abstract}

Index Terms-Coherent communications, orthogonal frequency-division multiplexing (OFDM), phase estimation.

\section{INTRODUCTION}

I $\mathrm{N}$ wireless communication systems, orthogonal frequencydivision multiplexing (OFDM) has been widely adopted in various standards, such as IEEE $802.11 \mathrm{~g}$. Recently, incoherent optical OFDM (IO-OFDM) [1], [2] and coherent optical OFDM (CO-OFDM) [3] have been independently proposed to combat chromatic dispersion in optical fiber. CO-OFDM has also been shown as the immunity to polarization-mode dispersion [4]. Furthermore, we have carried out a proof-of-concept experimental study on the feasibility of CO-OFDM [5]. However, the details of the phase estimation theory for CO-OFDM are not thoroughly discussed in [5]. Because coherent detection is employed in CO-OFDM, it is important to investigate the optical phase noise impact on system performance and explore the optimal approach to estimate phase evolution of OFDM signals. In contrast to conventional single carrier optical transmission systems, OFDM systems transmit a data stream over a number of lower rate subcarriers simultaneously. This opens an opportunity to employ advanced phase estimation techniques, such as pilot-aided phase estimation [6].

In this letter, we present the detailed theory and experiment of digital phase estimation with pilot subcarriers. We note the pilot-aided phase estimation has been studied in simulation to remove self-phase modulation in IO-OFDM [1]. However, it is the first time that pilot-aided phase estimation is experimentally demonstrated for optical OFDM transmissions. With pilot-aided phase estimation, we successfully reconstruct the quadrature phase-shift-keying (QPSK) constellations for CO-OFDM systems with a nominal data rate of $8 \mathrm{~Gb} / \mathrm{s}$ after 1000-km transmission over standard single-mode fiber (SSMF). The optical signal-to-noise ratio (OSNR) penalty is found to be

Manuscript received November 21, 2006; revised March 7, 2007

X. Yi and Y. Tang are with Victoria Research Laboratory, National ICT Australia, The University of Melbourne, Melbourne, VIC 3010, Australia (e-mail: x.yi@ee.unimelb.edu.au).

W. Shieh is with the ARC Special Research Centre for Ultra-Broadband Information Networks, Department of Electrical and Electronic Engineering, The University of Melbourne, Melbourne, VIC 3010, Australia.

Color versions of one or more of the figures in this letter are available online at http://ieeexplore.ieee.org.

Digital Object Identifier 10.1109/LPT.2007.897572

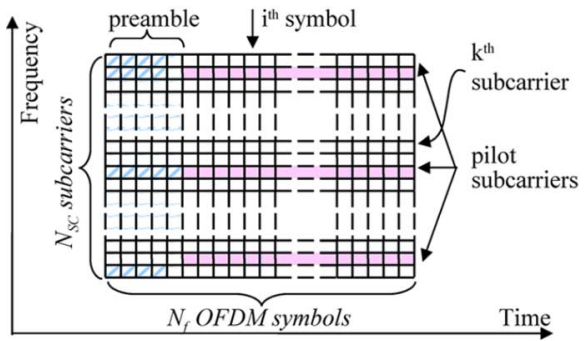

(a)

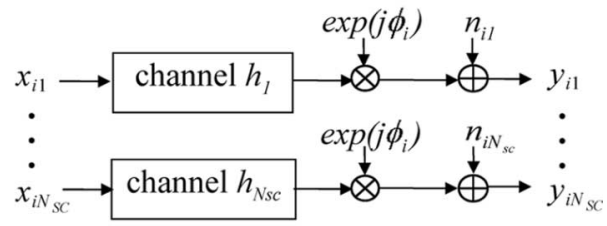

(b)

Fig. 1. (a) Two-dimensional time/frequency structure of one OFDM frame; (b) parallel subcarrier model for OFDM transmission.

about $1 \mathrm{~dB}$ at $10^{-3}$ bit-error rate (BER). The OSNR penalty degrades $0.5 \mathrm{~dB}$ by using data-aided phase estimation. We also show as few as five subcarriers are sufficient for pilot-aided phase estimation.

\section{TheORY OF PHASE ESTIMATION FOR CO-OFDM}

Fig. 1(a) shows the two-dimensional time/frequency structure for one OFDM frame, which includes $N_{f}$ OFDM symbols in time and $N_{\mathrm{SC}}$ subcarriers in frequency. The indexes of OFDM symbol and subcarrier are $i$ and $k$, respectively. The preamble is added at the beginning to realize frame synchronization and channel estimation. The frame synchronization follows the standard Schmidl format [6] and will not be discussed in this letter. A few subcarriers, i.e., pilot subcarriers, across OFDM spectrum are dedicated for phase estimation. This pilot-aided phase estimation takes advantage of the multiple parallel subcarriers in OFDM systems and would be difficult to be realized in conventional single carrier systems.

Phase noise upon OFDM signal reception after fast Fourier transform (FFT) consists of two components: a random noise term that can be modeled as additive Gaussian noise with zero-mean, and a common deterministic term originated from laser phase drift that affects all the subcarriers uniformly. The laser phase drift also causes intercarrier interference (ICI), which can be lumped into the random noise term [6] because a larger number of subcarriers are used in this work. The thrust of phase estimation is to extract laser phase drift from noisy received signals for OFDM subcarrier phase estimation and symbol decision. Because CO-OFDM utilizes linear RF-to-optical up conversion in the transmitter and linear optical-to-RF down conversion in the receiver [3], we model CO-OFDM transmission as parallel linear channels as shown in Fig. 1(b), 
where $x_{i k}$ and $y_{i k}$ are transmitted and received signal respectively, $h_{k}$ is the transfer function for the $k$ th subcarrier, $\Phi_{i}$ is the phase drift of the $i$ th OFDM symbol, and $n_{i k}$ is the white Gaussian noise with zero-mean. The transfer functions of the subcarriers in optical fibers are treated as static within one OFDM frame and obtained by using a training sequence in the preamble. Since the OFDM symbol used has a period of $36 \mathrm{~ns}$ and the laser sources both have about $20-\mathrm{kHz}$ linewidth in our experiment, the phase drift within one OFDM symbol can be considered as constant and common to all the subcarriers.

From Fig. 1(b), the channel model for the $k$ th subcarrier in the $i$ th OFDM symbol can be expressed as

$$
\begin{aligned}
y_{i k}= & x_{i k} \cdot h_{k} \cdot \exp \left(j \Phi_{i}\right)+n_{i k} \\
h_{k}= & \left|h_{k}\right| \cdot \exp \left\{j\left(\Phi_{0}+2 \pi \tau_{0} f_{k}+\frac{c \pi}{f_{\mathrm{LD}}^{2}} D_{t} f_{k}^{2}\right)\right\} \\
& i=1, \cdots, N_{f} ; k=1, \cdots, N_{\mathrm{SC}}
\end{aligned}
$$

where $h_{k}$ captures the group velocity delay from fiber chromatic dispersion, presumably a quadratic function of subcarrier frequency $f_{k}$. The group velocity delay consists of a zeroth-order dc term $\Phi_{0}$, a linear term proportional to the time delay of the first subcarrier $\tau_{0}$, and a quadrature term proportional to the fiber chromatic dispersion $D_{t}$ in the unit of picoseconds/picometers. $f_{\mathrm{LD}}$ is the optical carrier frequency.

As shown in (1), within the $i$ th OFDM symbol, the laser phase drift $\Phi_{i}$ is common to all the subcarrier but the Gaussian noise $n_{i k}$ is different for each subcarrier. Therefore, the first step of pilot-aided phase estimation is to compute the phase angle difference between the received and transmitted pilot subcarriers. Then the laser phase drift of each OFDM symbol is obtained by averaging the phase difference across all the pilot subcarriers. The estimated phase drift of the $i$ th OFDM symbol can be formally written as

$$
\Phi_{i}=\frac{1}{N_{p}} \sum_{k=1}^{N_{p}}\left\{\arg \left(y_{i k}\right)-\arg \left(\widehat{x}_{i k}\right)\right\}
$$

where $\arg ($.$) is the phase angle of the information symbol, \widehat{x}_{i k}$ is the known transmitted pilot subcarrier, and $N_{p}$ is the number of pilot subcarriers. After the $h_{k}$ and $\Phi_{i}$ are acquired, $x_{i k}$ in (1) can be estimated based upon the received symbol $y_{i k}$. The estimated $x_{i k}$, denoted as $\bar{x}_{i k}$, can be expressed as

$$
\begin{aligned}
\bar{x}_{i k} & =y_{i k} \cdot \exp \left(-j \Phi_{i}\right) \cdot h_{k}^{*} /\left|h_{k}\right|^{2} \\
i & =1, \cdots, N_{f} ; k=1, \cdots, N_{\mathrm{SC}}
\end{aligned}
$$

where $h_{k}^{*}$ is the conjugate of the estimated transfer function of the $k$ th subcarrier. The estimated transmit symbol $\bar{x}_{i k}$ is ready for OFDM symbol decision and BER calculation.

We define the phase estimation using data subcarriers as dataaided phase estimation. The channel model remains the same as (1). The $M$ th-power-law method is first used to remove the data modulation [7] and the residual phase angle consists of phase drift and Gaussian phase noise. After averaging across all the subcarriers in the $i$ th OFDM symbol, the estimated phase drift can be written as [3]

$$
\Phi_{i}=\frac{1}{N_{\mathrm{SC}}} \sum_{k=1}^{N_{\mathrm{SC}}}\left\{\arg \left(y_{i k}^{M}\right) / M\right\} .
$$

Similar to pilot-aided phase estimation, (4) is then used to obtain the $\bar{x}_{i k}$. Because of the phase ambiguity associated with the $M$ th-power-law method, the error of phase estimation by (5) can be significant when the phase noise is strong. Consequently, the estimated transmit symbol $\bar{x}_{i k}$ is not accurate. However, the accuracy of $\bar{x}_{i k}$ can be improved by iteration. A new $\Phi_{i}$ is estimated by substituting $y_{i k}$ with $\bar{x}_{i k}$ in (5). A new $\bar{x}_{i k}$ is then obtained in (4) by using the new $\Phi_{i}$.

The advantage of pilot-aided phase estimation is apparent in (3), namely, it does not suffer from phase ambiguity. Therefore, it can achieve accurate phase estimation even with a very noisy signal. On the other hand, conventional data-aided phase estimation suffers from phase ambiguity as a result of multiple levels of data signals. Differential encoders and decoders are generally added to resolve the phase ambiguity, but the effects from random noise cannot be completely avoided [7]. Therefore, the performance of data-aided phase estimation is degraded while its implementation complexity is increased because of the need for multiple-iteration of phase estimation.

\section{Phase Estimation for CO-OFDM AND Results}

We apply pilot-aided and data-aided phase estimation to a 1000-km CO-OFDM transmission experiment and the details of the experimental setup can be found in [8]. In the experiment, 128 QPSK modulated OFDM subcarriers are converted into time domain by inverse FFT (IFFT) in a Matlab program. The preamble and guard interval are subsequently inserted into one OFDM frame, which has 400 OFDM symbols as shown in Fig. 1(a). The resultant digital sequence is uploaded into an arbitrary waveform generator at $4 \mathrm{GSa} / \mathrm{s}$ to produce the real-time OFDM signals. A single Mach-Zehnder modulator biased at zero output is driven to produce 128 QPSK subcarriers with certain constraints [5], which corresponds to a bit rate of $8 \mathrm{~Gb} / \mathrm{s}$. Therefore, one OFDM symbol has a time duration of $36 \mathrm{~ns}$, including 4-ns guard interval. The transmission length in a recirculating loop is equivalent to $1000-\mathrm{km}$ transmission over SSMF. At the receiver side, the optical OFDM signal is coherently down-converted into the RF domain. The RF OFDM traces are acquired with a real-time sampling scope at $20 \mathrm{GSa} / \mathrm{s}$. The acquired traces are processed with another Matlab program as an OFDM receiver, which performs frame synchronization, channel estimation, phase estimation and compensation, and data decision. Pilot-aided and data-aided phase estimation are both realized according to the theory in Section II. In this experiment, the optical power into optical fiber is limited to about $-10 \mathrm{dBm}$ to avoid fiber nonlinearity, and the polarization of the receiver laser is manually aligned to the incoming optical signal. The nonlinearity and polarization diversity detection will be further investigated in our ensuing experiments.

Fig. 2 shows the phase estimation and compensation results for one OFDM frame after 1000-km transmission with $9.9 \mathrm{~dB}$ received OSNR. The bold curve (a) shows the result of pilotaided phase estimation, namely, the symbol phase drift $\Phi$ in (3). After phase compensation by (4), we can calculate the individual subcarrier phase noise as $\arg \left(\bar{x}_{i k}\right)-\arg \left(x_{i k}\right)$, illustrated by the bold curve (e). From the curve (a) and (e), the pilot-aided phase estimation only needs to be carried out once and the phase drift is fully compensated, which is demonstrated by the flat 


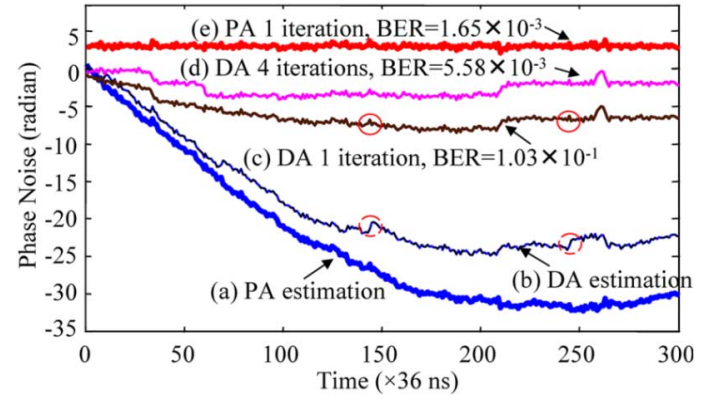

Fig. 2. OFDM symbol/subcarrier phase noise evolution versus time. The circles indicate the disappearance of the phase kinks after the phase compensation. PA: Phase-aided. DA: Data-aided.

phase noise evolution curve (e) only showing random noise. On the other hand, the symbol phase drift $\Phi$ of data-aided phase estimation in (5), as shown by the thin curve (b), clearly deviates from pilot-aided phase estimation, which is caused by phase ambiguity, particularly manifested as the occasional phase kinks. Similarly, we can calculate the individual subcarrier phase noise as $\arg \left(\bar{x}_{i k}\right)-\arg \left(x_{i k}\right)$ after phase compensation by (4). As a result of phase ambiguity, the phase noise evolution curve (c) shows residual phase drift and phase kinks. For the data-aided method, after one iteration of phase estimation and compensation, some phase kinks indicated by the small circles of the curve (c) are eliminated, which means less symbol decision errors. The residual phase drift can be further reduced for a better BER if we iterate phase estimation and compensation as shown by the curve (d). This is because the phase compensation of the last iteration reduces the BER and improves the phase estimation accuracy for the next iteration. We find four iterations are adequate to obtain the minimum BER since further iteration cannot improve phase estimation accuracy. However, this is still more complex and requires more computation power in comparison with pilot-aided phase estimation.

Although pilot subcarriers are effective for phase estimation, they have the disadvantage of consuming useful bandwidth. It is important to find the minimal number of pilot subcarriers to maintain sufficient performance. Fig. 3(a) shows the experimental BER performance as a function of number of pilot subcarriers at three different OSNRs after 1000-km transmission. It can be seen that five pilot subcarriers are adequate for the phase estimation because the BER performance is barely improved beyond five pilot subcarriers.

Based on the result in Fig. 3(a), we use five pilot subcarriers for CO-OFDM system performance evaluations. Fig. 3(b) shows the BER results of 1000-km transmission after applying the phase estimation. With pilot-aided phase estimation, the BER performance after transmission shows about 1-dB OSNR penalty at BER of $10^{-3}$. However, if data-aided phase estimation with four iterations is employed, the BER performance degrades over $0.5 \mathrm{~dB}$ either for the back-to-back or $1000-\mathrm{km}$ measurements in comparison with the pilot-aided phase estimation. Our approach using a small number of pilot subcarriers enhances the performance of CO-OFDM systems compared with data-aided phase estimation. This advantage will be more significant if we employ 8-ary PSK and beyond.

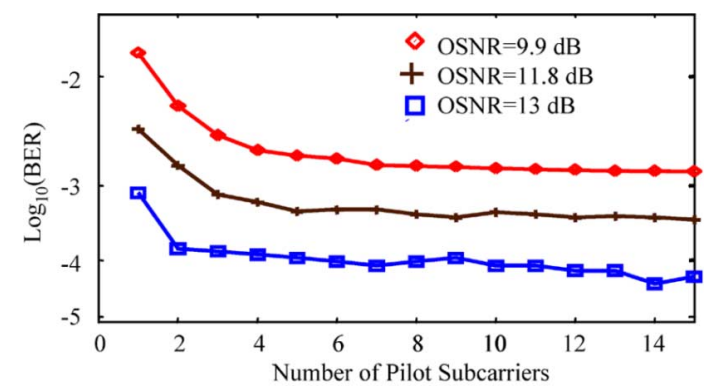

(a)

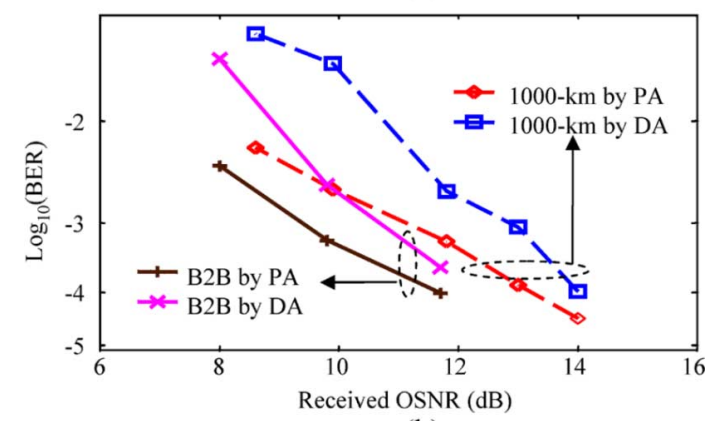

(b)

Fig. 3. (a) BER performance versus number of pilot subcarriers; (b) BER performance of 1000-km transmission. DA: data-aided, PA: pilot-aided, B2B: back-to-back.

\section{CONCLUSION}

In this letter, we have presented phase estimation and compensation methods for a CO-OFDM transmission experiment with a nominal data rate of $8 \mathrm{~Gb} / \mathrm{s}$ over 1000-km SSMF without optical dispersion compensation. The OSNR penalty is found to be about $1 \mathrm{~dB}$ at BER of $10^{-3}$. By using pilot-aided phase estimation, the system performance can be improved over $0.5 \mathrm{~dB}$ in comparison with data-aided phase estimation. We have also shown that as few as five pilot subcarriers are sufficient for pilot-aided phase estimation.

\section{REFERENCES}

[1] I. B. Djordjevic and B. Vasic, "Orthogonal frequency division multiplexing for high-speed optical transmission," Opt. Express, vol. 14, no. 9, pp. 3767-3775, May 2006.

[2] A. J. Loweiy, L. Du, and J. Armstrong, "Orthogonal frequency division multiplexing for adaptive dispersion compensation in long haul WDM systems," presented at the Conf. Optical Fiber Communication (OFC), USA, Mar. 2006, Paper PDP39.

[3] W. Shieh and C. Athaudage, "Coherent optical orthogonal frequency division multiplexing," Electron. Lett., vol. 42, no. 10, pp. 587-589, May 2006.

[4] W. Shieh, W. Chen, and R. S. Tucker, "Polarization mode dispersion mitigation in coherent optical orthogonal frequency division multiplexed syslems," Electron. Lett., vol. 42, no. 17, pp. 996-997, Aug. 2006.

[5] W. Shieh, X. Yi, and Y. Tang, "Experimental demonstration of transmission of coherent optical OFDM systems," presented at the Conf. Optical Fiber Communication (OFC), USA, Mar. 2007, Paper OMP2.

[6] L. Hanzo, M. Münster, B. Choi, and T. Keller, OFDM and MC-CDMA for Broadband Multi-User Communications, WLANs and Broadcasting. New York: Wiley, 2003.

[7] J. G. Proakis, Digilal Communications, 4th ed. Boston, MA: McGraw-Hill, 2000, ch. 5.

[8] W. Shieh, X. Yi, and Y. Tang, "Transmission experiment of multi-gigabit coherent optical OFDM systems over $1000 \mathrm{~km}$ SSMF fibre,' Electron. Lett., vol. 43, no. 3, pp. 183-184, Feb. 2007. 